\title{
DESIGN FOR RELIABILITY OF THE CONSTRUCTION ROBOT USING GENETIC ALGORITHM
}

\section{A.M. McCrea ${ }^{1}$ and G.J. Bleakley ${ }^{2}$}

${ }^{\prime}$ Ph.D. Candidate and Research Fellow - Robotics Unit, City University; Northampton Square, London ECI.

Research Assistant - South Bank University, ESD Dept., Borough Rd. London SEI OAA, UK.

Tel: ++44 - (0)171-477 8144; Fax: ++44 - (0)171-4778570;

E-mail:A.McCrea@city.ac.uk

${ }^{2}$ Ph.D. Candidate - Robotics Unit, City University, Northampton Square,

\section{London ECl.}

Lecturer - South Bank University, ESD Dept., Borough Rd. London SEI

$O A A, U K$.

Tel: ++44 - (0)171-815-8292; Fax: ++44 - (0)171-815-7699;

E-mail: G.Bleaklev@sbu.ac.uk

Abstract: Construction robot design for reliability is of particular importance due to the robots interaction with the environment and high level of human involvement, which impose additional safety hazards. Many methods, tools and approaches exist, however none offers full and exhaustive reliability analysis of the given system. Research presented in this paper links the dynamic modelling of a system (using VisSim software) with Genetic Algorithm as a search and optimisation tool to increase effectiveness of the reliability assessment, in a reduced time. The combined model is capable of analysing a complex system. for combinations of failure modes in combinations of components and sub-systems, which lead to system failure, in a manageable time. This in turn allows the system to be re-designed. to avoid the prospective risk of malfunction or undetected failure.

Keywords: Design for Reliability. Dynamic Modelling, Genetic Algorithms, Construction Robot.

\section{INTRODUCTION}

Design for safety is becoming an increasingly important aspect of the product design process. This concern is particularly applicable to mechatronics, as the variety of applications and innovative designs are increasing. Robots and automated devices designed and operating in the construction industry are of particular consideration, because the robot's working environment is constantly interacting with unstructured human involvement, and any malfunction poses an additional health hazard [12]. 
Failure analysis is carried out using tools, which fall into three groups: qualitative, quantitative and computer based modelling tools. Qualitative methods apply various fault scenarios to the system and analyse the effects of failure propagation through the system. Needless to say, these methods do not search all possible combinations of failure scenarios in all elements or sub-systems. Quantitative tools deal with determining the system characteristics from the mathematical models of the system. The result of applying such methods is an analysis of the probability of failure of the system. The computerbased modelling tools are the closest to attempt to investigate and design out failure scenarios from the system, in a methodical manner. The modelling tools can either be expert-based, state behaviour mathematical models, or dynamic simulation models.

This research proposes the use of the combination of dynamic modelling using VisSim software and Genetic Algorithms (GA). VisSim is used to create a dynamic model of the system with integrated failure modes, GA are used as an optimisation tool to search for the most likely failure scenarios under pre-determined conditions.

\section{APPROACH TO RELIABILITY AND DESIGN FOR RELIABILITY}

Design for safety and reliability is applied to a robot, which uses a Gamma radiography NDT probe, a description of this robot can be found in Chamberlain et. al, [5]. Because of the highly hazardous nature of Gamma radiation it is necessary to know the position of the Gamma source at all times, and prevent its movement, when a malfunction of the system has occurred. Therefore, the failure conditions are set as: (i) Gamma source fails to stop within $0.06 \mathrm{sec}$ of when the failure is detected by the computer model, or (ii) the position of Gamma source is not known to within $1 \mathrm{~mm}$ of its actual position $0.06 \mathrm{sec}$ after a failure has been detected by the computer model.

Because of the mechatronic nature of the system under investigation, much of the reaction to failure is built into the robot controller. The aim of safe design. in this instance, is the scenario when the controller recognises a fault in the system and implements system shutdown, which leaves the system in a 'SAFE' condition, within a certain sets of constraints (see previous paragraph). However, if the system is still operating after the constraints are violated, under the fault conditions, this indicates that the safety is infringed, the system is malfunctioning and needs redesigning. as the fault-condition is not observed by the controlling device. Components and sub-systems in a system can fail in several ways. These faults are called modes of failure and a sub-system or component can have many modes of failure although they may only reflect few differences in failure behaviour,

Two qualitative methods require special attention, as using them prior to and post VisSim modelling of a robot allows a more guided approach to failure analysis. Failure Modes Effects Analysis (FMEA) [I] is carried out initially, in order to identify possible failure modes. FMEA is a bottomup tool for evaluating the various modes of subsystem or component failure in terms of (i) likelihood of their occurrence, (ii) their effect on the complete system, and (iii) and how detectable they are. The higher the probability of occurrence or severity, the higher value is allocated to the measuring factor. The increasing detectability attracts a decreasing value of the factor. The overall level of risk is recognised as the product of all three factors, which results in Risk Priority Number (RPN). The mode of failure achieving the highest RPN tends to represent the worst case of failure. Using the results of the analysis, solutions are investigated to counter the causes or effects of each failure mode. However, the components failures are only considered as separate entities, how a failure in 
one component influences other elements is often ignored and the process reflects a static analysis of the system.

The objective of using the VisSim model is to take into account the effects of failure propagation and dynamic failure effects within the system.

VisSim is a block diagram language for mathematical modelling and simulation, which supports both linear and non-linear systems. It allows the dynamic and logic modelling of software, electronic hardware and the mechanical components of a system to be carried out. Failure functions are added as alternative behaviours to the components or sub-systems. The software is capable of switching within the model, the functionality of the component from correct behaviour to the failure behaviour (see Figure 1, below).

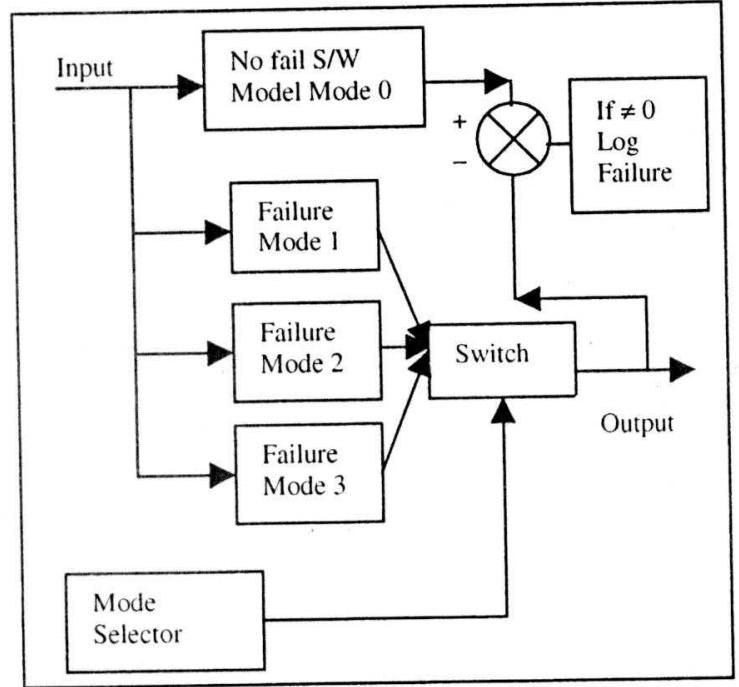

Figure 1. Schematic of Software

Implementation of Failure Mode Detection.

By selecting particular failure modes [4], running a sequence of operations and then comparing the behaviour of the perfect functionality to the failure functionality, it is possible to determine which failure modes cause the system to fail. When any of the failure criteria are met, the failure mode and component are logged in a failure file for further reference. The identification of the critical elements in critical locations within the system implies the need to revisit, redesign or relocate in order not to lead to failure of the complete system.

The final stage leads to identification of the components most likely to cause failure. This can be achieved through external critical analysis of VisSim generated results. And here the second major qualitative tool has to be employed - Fault Tree Analysis (FTA) [1]. Fault Tree Analysis is a systematic way of identifying all possible modes of failure of all possible elements and sub-systems. It is a 'top-down' method, which shows hierarchically logical combinations of faults and conditions that can cause the fail-danger failure. Faults which lead to further faults are joined by AND or OR logic gates. An AND gate is used when two or more sub-faults must be present to produce a fault. OR gates are used when either of two or more sub-faults is required to produce a system failure. The system can be analysed further, by determining minimum cut sets, which are the minimum series of events leading to a system failure. The worst scenario is a system. which has a minimum cut set consisting of a large number of single-point failures. A single point failure (SPF) is identified as a failure of a single component, which leads to the failure of the whole system. Double point failure (DPF) is specified as a failure of the system caused by combined failure of two components. Meaning of the triple and quadruple point failures can be deducted from above definitions.

The complex system analysis produces a very large fault tree due to the amount of components. sub-systems and their combinations of failure modes. This fault tree has to be analysed to determine the minimum cut sets and the Reduced Fault Tree (RFT), which is the compilation of the minimum cut sets. Due to the size of the original fault tree and number of hierarchical levels this analysis presents an enormous task.

To reduce the fault tree in a logical, easy to apply manner, failure modes are selected in stages and the number of failure modes is increased at each stage. The combinations, which contain failure 
modes. which already have been identified. are removed from the failure selection file [3]. This dramatically reduces the number of failure mode combinations, which need to be investigated and simplifies the analysis of the results. From the data gathered in the failure file, it is possible to generate a RFT, thus identifying the components most likely to cause failure [2].

This general process of FMEA, system analysis and FTA provides the basis for many design methodologies for reliability.

\section{APPLICATION OF GENETIC ALGORITHMS}

A logical model and a dynamic model are necessary for the accurate fault analysis of a system. However, fault analysis based on a dynamic model's behaviour is not common due to its alleged excessive calculation timescale. The logical and hierarchical analysis of the contents and meaning of the failure file in order to obtain RTF, is one way of guiding the VisSim's performance, reducing the excessive computing time and putting the outcome in more manageable and understandable form. If however another method to obtain RFT could be found the high computation overhead could be dramatically reduced and the method could become viable.

Therefore, a powerful search and optimisation tool needs to be linked into the VisSim model, in order to reduce the run time by optimising and automating the search for the failure modes. The tool selected to carry out this task is Genetic Algorithm (GA).

\section{I Brief Overview of Generic Algorithms}

The adaptive search technique known as the Genetic Algorithm is an effective optimisation tool [9] and has been applied within the engineering design process for numerous applications. Here. the design for reliability has been used as an environment for GA application to a robot with NDT probe using Gamma radiography.

The basic principle of this technique is to translate a technical problem into a genetic one and optimise it, using the biological selection mechanisms of nature [7]. This method ensures, to a very high degree of likelihood, that the absolute optimum is found. GA proves to be a highly efficient method of optimisation, if used wisely, and an invaluable tool within the design process.

The first step towards any GA application is to define criteria for optimisation as a measurable quantity, which generally defines the problem in the broad sense [8]. Optimisation criteria can be defined directly or indirectly when the external, critical requirements, such as: task characteristics, functional desires, customers' requirements, etc., need to be translated into a technical language. At this stage boundary conditions for the parameters also have to be set. The second step focuses on establishing all the parameters significant to the required result and then ranking, selecting and evaluating them.

In order to apply GA all the parameters have to be represented and combined in special. coded strings, which become candidate solutions to the problem and are frequently called representations. In general terms, the representation of the design is a direct consequence of the choice of parameters for optimisation (as they characterise the design), while the criteria serve to evaluate the quality of the solution. The basis of the success of the GA is the fact that the 'system' under design or optimisation is represented as a model consisting of all the optimised parameters. A pool of such models is created (called - population), with the possible values of the parameters randomly varying. within the parameters' constraints. Each combination of the values of the parameters is assessed and evaluated using a fitness function. Fitness, or evaluation, calculations are carried out through an objective function. embracing 
the criteria. The whole process combines the following: (i) rejection of parameter combinations, which produce low values and hence, unfit designs. (ii) preferential reproduction of the more successful combinations and (iii) random generation of new values for further testing. All these operations enable the GA technique to sample varying areas of the design space whilst concentrating on highly fit regions. A detailed description of the structure and use of the GA technique may be found in Davis [6].

\subsection{GA Optimisation within the VisSim Performance}

The research approach described in section two falls well within the scope of GA, however simple GA are not well suited for fine-tuning optimisation. Therefore, a conventional heuristic, such as beam search is incorporated (hybridised) into GA. Beam search is a heuristic refinement of the breath first search method that relies on the notion of beam width' to restrict the number of nodes that are branched from at each stage. At each level of the search process, all nodes are evaluated using a predefined evaluation function, which is the failuremodelling result from VisSim. Then the beam width ' $w$ ' is used to pick ' $w$ ' amount of best nodes in terms of their evaluation to branch from at the current level, to generate the offspring nodes at the next level.

GA parameters within this search are identified as the modes of failure of all the elements. The components and sub-systems (genes) make up the string (chromosome) representing the model under investigation with all its elements and their modes of failure in a consecutive form. In the case study the number of failure modes varies from one to four per element. The evaluation is carried out according to the criterion of the optimisation, which is classified as the identification of the failure modes, which produce failure in the model according to pre-defined constraints. Evaluation of further elements in the population depends upon the success or failure of the members of population to produce a failure. The initial population contains a SPF in every member of the population. The results of the VisSim simulation of every member of the population for SPF identify all 'failure SPF' and result in the beam width ' $w$ ', encompassing all the SPF, which do not produce the system failure. The 'failure SPF' are pruned out permanently and their future presence in the population is penalised in terms of the chromosome's evaluation. From each of the remaining ${ }^{\prime} w$ ' nodes. all possible successor nodes are generated. The VisSim evaluation of this stage produces all DPF. which are eliminated for the next level beam search. Subsequent generations evolve into higher point failures around critical failure modes at each level.

An additional parameter is added at the later stage, this results from VisSim's ability to calculate the time to failure, this can be used as a measure of the degree of failure. The degree of failure is identified as the time taken for the system to recognise the failure and react appropriately. Subsequently, the degree of failure is reflected in the fitness value.

The final aim of the optimisation is a series of chromosomes representing the minimum cut sets of failure modes at each level. From this a RFT can be generated.

\subsection{Combining GA and VisSim}

VisSim models, when originally applied to the failure analysis problem were represented in a graphical format on the computer screen, the upkeep of this graphical model is very computer intensive. In order to reduce the time spent on generating the image and to enable the model to be linked with GA software it is necessary to change the model to $\mathrm{C}$ or $\mathrm{C}++$ code. This is achieved using the VisSim $\mathrm{C}$ code generator linked with the original VisSim software. C VisSim models run five times faster than equivalent graphical models [11]. This enables more 
complex models to be produced with little computational overhead and increases the speed of the analysis.

The GA code is created in $\mathrm{C}++$ to link in with the VisSim C code model and because there are many public domain GA programs already available with libraries of functions [10]. These libraries are extended by creating customised software for this application using Visual $\mathrm{C}++6$.

\section{CONCLUSIONS}

The research presented in this paper enables the design for reliability of complex systems to be carried out efficiently, within pre-set safety constraints. It uses existing dynamic modelling software VisSim and incorporates the outcome assessment and evaluation within its coding using GA as the search and optimisation tool. Dynamic modelling of a system is superior to any other tool used for the assessment for failure, due to the detail in which the analysis is carried out. So far the scale of the computations, the randomness and the necessity to assess the outcome manually, were the main obstacles in using existing software for reliability design. Using $\mathrm{GA}$ as internally linked search and optimisation tool, to guide the VisSim's choice of the modes of failure and choice of components and sub-systems and then to assess the performance makes this combined model a practical and efficient tool for reliability analysis.

\section{REFERENCES}

[1] Bentley, J.P. (1999). Reliability \& Quality Engineering, Addison-Wesley, $2^{\text {nd }}$ Ed.

[2] Bleakley, G.J. (1999). "Combining Computerised Failure Analysis with EQFD", Proceedings of
International Conference on Engineering Design. ICED 99, Munich, August 1999.

[3] Bleakley G.J., Edney, R.C \& Chamberlain D.A., (1997) "The Use Of DFD And ACP Diagrams. For Mechatronics Systems Design", International Conference On Engineering Design, ICED 97

Tampere, August 19-21, 1997, Vol. 3.

[4] Chamberlain D., Bleakley G. (1998) "Design Methodology for Mechatronic Systems". DoE. City University, Construction Robotics Unit.

[5] Chamberlain D.A., Bleakley G.J., Edney R.C. (1996), "Robotic Handling of Gamma Ray Sources in Site Radiography of Steel Storage Tanks", 13th. International Symposium on Automation and $\underline{\text { Robatics in Construction, Elsevier. }}$

[6] Davis, L. ed. (1991). Handbook of Genetic Algorithms, New York: Van Nostrand Reinhold. USA.

[7] Goldberg, D.E. (1989). Genetic Algorithms in Search, Optimisation and Machine Learning. Reading, MA: Addison-Wesley, UK.

[8] Krottmaier, J. (1993). Optimising Engineering Designs, McGraw-Hill Book Company Europe. London, UK.

[9] Mitchell, M. (1996). Introduction to Genetic Algorithms, A Bradford Book, The MIT Press, USA.

[10] Michalewicz, Z. (1992). Genetic Algorithms + Data Structures $=$ Evolution Programs, Germany: Springer - Verlag.

[11] VisSim (1999). Visual Solutions Incorporated. VisSim C-Code, Software Manual.

[12] Zhang L.,(1996), "Sensory Safety Systems for Robotic Construction Plant", Draft M.Phil. Thesis. Lancaster University, 1996. 